\title{
Association between aberrant dynein cytoplasmic 1 light intermediate chain 1 expression levels, mucins and chemosensitivity in colorectal cancer
}

\author{
CHUN-CHAO CHANG ${ }^{1,2^{*}}$, KUO-CHING CHAO ${ }^{1,2^{*}}$, CHI-JUNG HUANG ${ }^{3-5}$, \\ CHIH-SHENG HUNG ${ }^{6}$ and YEN-CHIEH WANG ${ }^{5,7}$
}

\begin{abstract}
${ }^{1}$ Division of Gastroenterology and Hepatology, Department of Internal Medicine, Taipei Medical University Hospital, ${ }^{2}$ Division of Gastroenterology and Hepatology, Department of Internal Medicine, School of Medicine, College of Medicine, Taipei Medical University, Taipei 11031; ${ }^{3}$ Department of Medical Research, Cathay General Hospital, Taipei 10630; ${ }^{4}$ Department of Biochemistry, National Defense Medical Center, Taipei $11490 ;{ }^{5}$ School of Medicine, College of Medicine, Fu Jen Catholic University, New Taipei $24205 ;{ }^{6}$ Division of Gastroenterology, Department of Internal Medicine;

${ }^{7}$ Division of Urology, Department of Surgery, Cathay General Hospital, Taipei 10630, Taiwan, R.O.C.
\end{abstract}

Received September 24, 2019; Accepted March 19, 2020

DOI: $10.3892 / \mathrm{mmr} .2020 .11086$

\begin{abstract}
Dynein transport along the cytoskeletal microtubules towards the minus end is essential for cell division, cell migration and other basic cellular functions. Dynein cytoplasmic 1 light intermediate chain 1 (DYNC1LI1) has been previously associated with pancreatic ductal adenocarcinoma, hepatocellular carcinoma and prostate cancer. Cytoskeletal structures are involved in the regulation of the mucosal barrier integrity. Thus, improving our understanding of the molecular mechanisms that regulate the mucosal barrier is critical for cancer management and treatment. The present study aimed to investigate DYNC1LI1 expression in colorectal cancer (CRC) tissues. The American Joint Committee on Cancer Stage II CRC cell line LS 174T was used to determine the association between the cellular expression levels of DYNC1LI1 and different types of mucin (MUC) by reverse transcription-quantitative PCR. The role of DYNC1LI1 in cell chemosensitivity and proliferation was also evaluated in the presence of the DNA analog 5-fluorouracil (5-FU) or the platinum-based
\end{abstract}

Correspondence to: Dr Yen-Chieh Wang, Division of Urology, Department of Surgery, Cathay General Hospital, 280 Ren'ai Road, Taipei 10630, Taiwan, R.O.C.

E-mail: ycwangurology@gmail.com

${ }^{*}$ Contributed equally

Abbreviations: DYNC1LI1, dynein cytoplasmic 1 light intermediate chain 1; CRC, colorectal cancer; MUC, mucin; RT-qPCR, reverse transcription-quantitative PCR; 5-FU, 5-fluorouracil; shRNA, short hairpin RNA

Key words: dynein cytoplasmic 1 light intermediate chain 1, mucin, colorectal cancer, 5-fluorouracil, oxaliplatin drug, oxaliplatin by the MTT assay. LS 174T cells with decreased expression levels of DYNC1LI1 were discovered to be more sensitive to 5-FU compared with LS 174T cells with endogenous DYNC1LI1 expression levels. Moreover, LS 174T cells transfected with short hairpin RNA targeting DYNC1LI1 were associated with low MUC1 and high MUC2, MUC4 and MUC5AC expression levels. Notably, the CRC cells with low MUC1 expression levels and high expression levels of the other MUCs (MUC2, MU4 and MUC5AC) were shown to benefit from 5-FU treatment. In conclusion, the findings of the present study have suggested that DYNC1LI1 expression may be significantly associated with MUC expression levels and may be used to predict the chemotherapeutic efficiency. However, additional functional studies and clinical reports are required for an improved understanding of the significance of these molecular interactions in tumorigenesis.

\section{Introduction}

Cytoskeletal rearrangement is required for cell migration and invasion, which are also important steps for the process of cancer metastasis $(1,2)$. Dynein transport along the cytoskeletal microtubules towards the minus end is essential for cell division, migration and cytoskeletal motors (3-5).

The cytoplasmic dynein complex is implicated in intracellular vesicular transport (6). Numerous studies have reported that microtubule-dependent vesicular trafficking serves a central role in cell function and may impair tumor invasion (7-9). The expression levels of cytoplasmic dynein, particularly dynein light chains, have been associated with several types of cancer in humans; for example, melanoma cell apoptosis was associated with dynein light chain LC8-type (DYNLL)1 and DYNLL2 expression (10); and upregulation of dynein light chain roadblock-type (DYNLRB)1 and downregulation of DYNLRB2 expression levels were detected in hepatocellular carcinoma (HCC) (11). Moreover, in another study investigating HCC, aberrant expression levels of dynein 
cytoplasmic 1 light intermediate chain 1 (DYNC1LI1) were observed (12). DYNC1LI1 expression has also been detected in the urine of patients with pancreatic ductal adenocarcinoma (13), and increased DYNC1LI1 phosphorylation was also found in prostate cancer $(\mathrm{CaP})(14)$. Altogether, these findings suggested that DYNC1LI1 may serve a potential tumorigenic role in numerous types of human cancer.

The epithelia in organ systems, such as the urinary and gastrointestinal systems, are protected from pathogenic infections by a mucosal barrier $(15,16)$. Mucin (MUC) expression is critical for the formation of the mucosal barrier (17). Two functionally distinct classes of MUCs, secretory gel-forming MUCs and transmembrane MUCs, have been identified in different epithelial cells $(18,19)$. Both MUCs have been discovered to be intimately involved in the inflammatory response and tumorigenesis (19). In addition, the cytoskeletal structures have also been found to be involved in the regulation of the mucosal barrier integrity and its breakdown during inflammation (20). Therefore, molecular detection methods that can improve our understanding of the mucosal barrier are critical to both cancer management and treatment. In the present study, the expression levels of DYNC1LI1 were investigated in colorectal cancer (CRC) tissues and in the human epithelial colon cell line LS 174T, which is considered by the American Joint Committee on Cancer (AJCC) to be a model of stage II CRC and to express different MUCs (21). A series of reverse transcription-quantitative PCR (RT-qPCR) experiments were performed to determine the association between the expression levels of DYNC1LI1 and different MUCs in LS $174 \mathrm{~T}$ cells.

\section{Materials and methods}

Cell lines and reagents. The goblet-cell-like CRC cell line, LS 174T (AJCC stage II; CL-188) and two other CRC stage II cell lines, SW480 (CCL-228) and HCT 116 (CCL-247), were purchased from the American Type Culture Collection. The clinical status of the cell lines and the culture conditions are available on the ATCC website (www.atcc.org). Briefly, LS 174T cells were cultured in minimum essential medium (cat. no. 41500-034; Thermo Fisher Scientific, Inc.), HCT 116 cells were cultured in DMEM (cat. no. 12800-017; Thermo Fisher Scientific, Inc.) and SW480 cells were cultured in L-15 medium (cat. no. 41300-039; Thermo Fisher Scientific, Inc.). All the culture mediums were supplemented with $10 \%$ fetal calf serum (cat. no. A6806-35; NQBB International Biological Corp.) and 1\% antibiotic/antimycotic solution (cat. no. 15240-062; Thermo Fisher Scientific, Inc.). LS 174T cells and HCT 116 cells were maintained in a humidified chamber with $95 \%$ air and $5 \% \mathrm{CO}_{2}$. SW480 cells were maintained in a humidified incubator with $100 \%$ air at $37^{\circ} \mathrm{C}$. The Human Reference cDNA (HRC; cat. no. 636692; Takara Bio, Inc.) was used as an expression control for DYNC1LI1.

Lentiviral knockdown of DYNC1LI1. The lentiviral construct, pLKO_TRC005-DYNC1LI1 (clone ID: TRCN0000299843), with short hairpin RNA (shRNA) to target DYNC1LI1 (shDYNC1LI1), was used to knockdown the expression level of DYNC1LI1. The control pLKO_TRC005-luciferase (LUC; clone ID: TRCN0000231719) vector targeting LUC was used as the negative control (shLUC-LS 174T) for the shDYNC1LI1. Both the lentiviral constructs were obtained from the RNA Technology Platform and Gene Manipulation Core (Sinica, Taiwan). A total of $1.25 \times 10^{5} \mathrm{LS} 174 \mathrm{~T}$ cells/well were cultured in a 6-well plate for $24 \mathrm{~h}$ and the subsequent lentiviral infections (multiplicity of infection=3) were performed to knock down the expression of DYNC1LI1 in LS 174T cells (shDYNC1LI1-LS 174T). Subsequently, medium containing $2 \mathrm{mg} / \mathrm{ml}$ puromycin (Thermo Fisher Scientific, Inc.) was used to select for and maintain stable clones. Following incubation for $48 \mathrm{~h}$, the infection efficiency of DYNC1LI1 knockdown in LS 174T cells compared with the shLUC-LS 174T cells was determined using RT-qPCR and western blotting.

Western blotting. Total protein was extracted from shLUC-LS 174T and shDYNC1LI1-LS174T cells using the PRO-PREP ${ }^{\mathrm{TM}}$ Protein Extraction solution (Intron Biotechnology, Inc.) according to the manufacturer's protocol, supplemented with a protease inhibitor (cat. no. P8340; Merck KGaA, Inc.). Total protein was quantified using Bio-Rad Protein Assay reagent (cat. no. 500-0006; Bio-Rad Laboratories, Inc.) and $15 \mu \mathrm{g}$ protein/lane was denatured in $1 \mathrm{X} \mathrm{NuPAGE}^{\mathrm{TM}}$ LDS sample buffer (Thermo Fisher Scientific, Inc.) for $10 \mathrm{~min}$ at $95^{\circ} \mathrm{C}$, and separated via $12 \%$ SDS-PAGE. The separated proteins were subsequently transferred onto a PolyScreen 2 PVDF Transfer membrane $(0.2 \mu \mathrm{m}$; PerkinElmer, Inc.). The membranes were blocked with $3 \%$ bovine serum albumin (cat. no. ALB001.100; BioShop Canada Inc.) for $1 \mathrm{~h}$ at room temperature and incubated with the following primary antibodies for $1 \mathrm{~h}$ at room temperature: Anti-DYNC1LI1 (1:1,000; cat. no. ab154251; Abcam) and anti- $\beta$-actin (1:1,000; cat. no. MAB1501R; Merck $\mathrm{KGaA}$ ). Following the primary antibody incubation, the membranes were washed with 1X TBST wash buffer $(20 \mathrm{mM}$ Tris, $0.15 \mathrm{M} \mathrm{NaCl}, 0.1 \%$ Tween-20; $\mathrm{pH} 7.4$ ) three times (15 min/ time) at room temperature. Subsequently, the membranes were incubated with an alkaline phosphatase-conjugated anti-rabbit antibody (1:5,000; cat. no. A3812; Merck KGaA) or alkaline phosphatase-conjugated anti-mouse antibody (1:5,000; cat. no. A9316; Merck KGaA) for $60 \mathrm{~min}$ at room temperature. Protein bands were visualized using a VECTASTAIN ${ }^{\circledR}$ ABC-AmP DuoLuX chemiluminescent/fluorescent substrate kit (cat. no.AK-6000; Vector Laboratories, Inc.), according to the manufacturers' protocol, and a FluorChem FC2 system (Cell Biosciences, Inc.).

$C R C$ cDNA array and RT-PCR. DYNC1LI1 expression levels were quantified using a cDNA array of colonic tissues covering four CRC stages $(n=40)$ and normal colonic tissues (n=8; cat. no. HCRT104; OriGene Technologies, Inc.). Total RNA from the cultured cells was extracted using the Easy Pure Total RNA Mini kit (Bioman Scientific Co. Ltd.), according to the manufacturer's protocol. Subsequently, total RNA (1 g) was reverse transcribed into single-stranded cDNA using an oligo(dT)12 primer with a High Capacity cDNA Reverse Transcriptase kit (cat. no. 4368813; Thermo Fisher Scientific, Inc.), according to the manufacturer's protocol. DYNC1LI1 and MUC2 mRNA expression levels were subsequently quantified by qPCR using the TaqMan Master mix (Roche Diagnostics GmbH). The following thermocycling 
Table I. Primers and universal TaqMan probes used for the reverse transcription-quantitative PCR.

\begin{tabular}{lll}
\hline Gene number $^{\mathrm{a}}$ & NCBI reference sequence & \multicolumn{1}{c}{ Primer sequence $\left(5^{\prime} \rightarrow 3^{\prime}\right)$} \\
\hline $\begin{array}{l}\text { Dynein cytoplasmic 1 light } \\
\text { intermediate chain 1 }\end{array}$ & NM_016141 & F: CTGGTGTGAGTGGTGGTAGC \\
Mucin 2 & NM_002457 & R: TCTGCATGAACATCTAAGACAGG \\
& NM_002046 & F: ATGCCAGCATTTGCATCC \\
GAPDH & & F: CTCTGCTCCTCCTGTTCGAC \\
& R: ACGACCAAATCCGTTGACTC
\end{tabular}

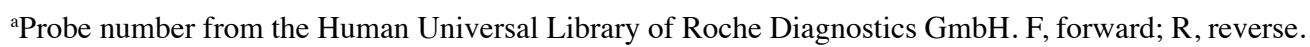

conditions were used for qPCR: Initial denaturation at $95^{\circ} \mathrm{C}$ for $10 \mathrm{~min}$; followed by 60 cycles of $95^{\circ} \mathrm{C}$ for $10 \mathrm{sec}$ and $60^{\circ} \mathrm{C}$ for $20 \mathrm{sec}$ (22). The primer pairs and universal TaqMan probes used for the qPCR are presented in Table I. Expression levels of MUC1, MUC4, MUC5AC and MUC6 were determined as previously described by Ohuchida et al (23). Expression levels were quantified using the $2^{-\Delta \Delta C q}$ method and normalized to the internal reference gene GAPDH.

Determining the chemosensitivity and proliferative rate of shLUC-LS $174 T$ and shDYNC1LI1-LS $174 T$ cells. The chemosensitivity and proliferative rate of shLUC-LS 174T and shDYNC1LI1-LS 174T cells were determined using a MTT assay (cat. no. M5655; Sigma-Aldrich; Merck KGaA). Briefly, $3 \times 10^{3}$ shLUC-LS $174 \mathrm{~T}$ or shDYNC1LI1-LS $174 \mathrm{~T}$ cells/well were cultured in 96-well plates for overnight at $37^{\circ} \mathrm{C}$ before drug treatments. The chemosensitivity of cells to the DNA analog 5-fluorouracil (5-FU; Merck KGaA) or the platinum-based drug oxaliplatin (Merck KGaA) at various concentrations $(0.01 \mu \mathrm{M}$ to $37.5 \mathrm{mM}$ for $5-\mathrm{FU}$ and $2 \mathrm{nM}$ to $1,000 \mu \mathrm{M}$ for oxaliplatin) was plotted after $72 \mathrm{~h}$ of incubation at $37^{\circ} \mathrm{C}$ and the half-maximal inhibitory concentration $\left(\mathrm{IC}_{50}\right)$ for each compound was calculated using Gen5 $5^{\mathrm{TM}}$ Data Analysis (version 2.04; BioTek Instruments, Inc.).

To determine the cell proliferative rate, $5 \times 10^{3}$ shLUC-LS 174T and shDYNC1LI1-LS174T cells/well were plated into 96-well plates and incubated alone, or with the $\mathrm{IC}_{50}$ dose of 5-FU or oxaliplatin for the 1-7 days at $37^{\circ} \mathrm{C}$.

For both assays, the cells were treated with $10 \mu \mathrm{l}$ MTT reagent in the dark for $4 \mathrm{~h}$ at room temperature and then $100 \mu \mathrm{l}$ DMSO was added/well to dissolve the purple formazan formed by the live cells. The absorbance at $540 \mathrm{~nm}$ of each well was measured using a Synergy HT Multi-Mode microplate reader (BioTek Instruments, Inc.). Data were obtained from three independent experiments.

Statistical analysis. Statistical analysis was performed using SPSS version 15.0 software (SPSS, Inc.). Statistical differences between 2 groups were determined using a Student's t-test, whereas statistical differences between $>2$ groups were determined using a one-way ANOVA, followed by a Bonferroni's post hoc test for multiple comparisons. Data was presented as the mean $\pm \mathrm{SD}$. $\mathrm{P} \leq 0.05$ was considered to indicate a statistically significant difference.

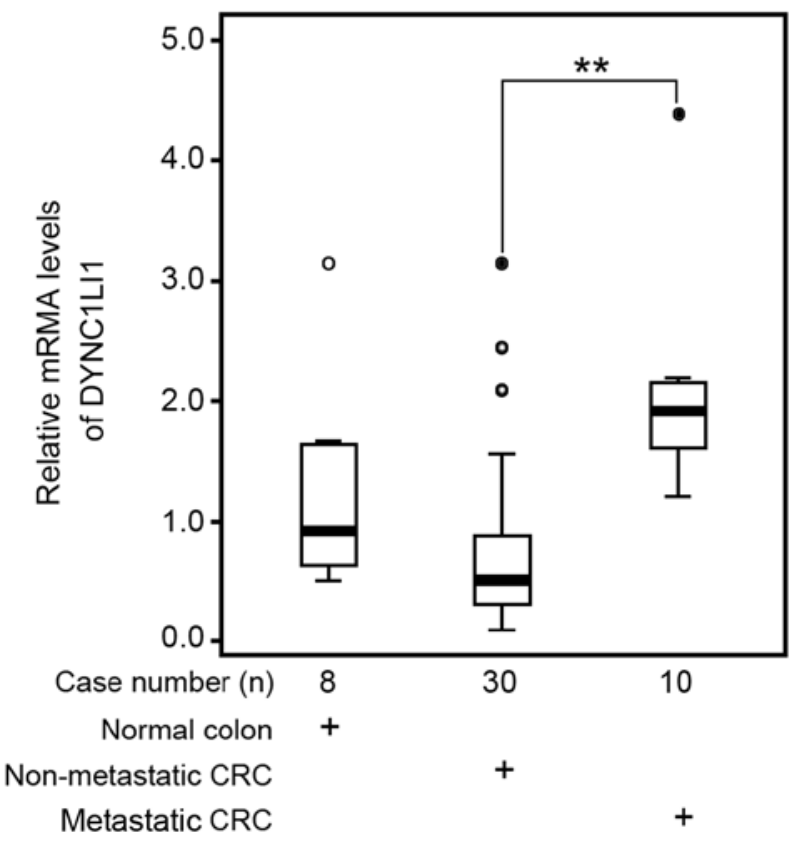

Figure 1. DYNC1LI1 expression levels in CRC tissues. The differential expression levels of DYNC1LI1 in normal and tumor colonic tissues from a CRC cDNA array were determined using quantitative PCR. Gene expression was normalized to GAPDH. The solid black line denotes the median for each group and the box depicts the 25 and 75 th percentile ranges. Capped error bars represent the $95 \%$ range of expression levels. Circles represent outliers and solid circles represent extreme values. Data were analyzed using a one-way ANOVA, followed by Bonferroni's post hoc test. ${ }^{* *} \mathrm{P}<0.01$. DYNC1LI1, dynein cytoplasmic 1 light intermediate chain 1; CRC, colorectal cancer.

\section{Results}

DYNCILI1 expression levels in CRC tissues. The differential expression levels of DYNC1LI1 in CRC were analyzed in cDNA arrays of colonic tissues containing normal colonic tissues and four CRC stages. DYNC1LI1 expression levels were significantly increased in cDNA samples from patients with metastasis $(n=10)$ compared with the patients without metastasis ( $=30 ; \mathrm{P}<0.01$; Fig. 1$)$.

Differential MUC expression levels in DYNC1LI1 knockdown LS $174 T$ cells. DYNC1LI1 mRNA expression levels were quantified in three CRC cell lines: HCT 116, SW480 


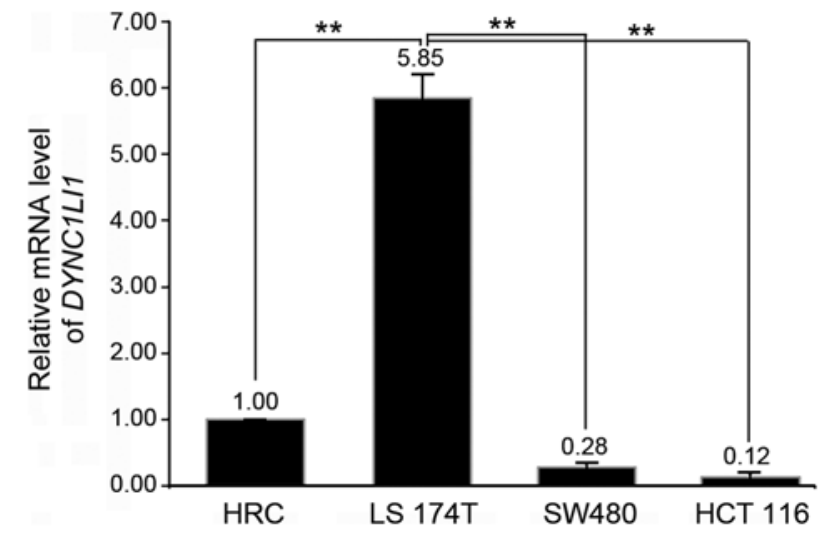

Figure 2. Relative expression levels of DYNC1LI1 in CRC cell lines. DYNC1LI1 mRNA expression levels were determined in three CRC cell lines: HCT 116, SW480 and LS 174T. The expression levels of DYNC1LI1 in CRC cell lines were determined using reverse transcription-quantitative PCR. All mRNA levels were normalized to the expression levels of GAPDH and are relative to that of HRC. Experiments were independently performed in duplicate or triplicate. Data were analyzed using a one-way ANOVA, followed by Bonferroni's post hoc test. Numerical data are expressed as the mean $\pm \mathrm{SD}$. ${ }^{* *} \mathrm{P}<0.01$. HRC, human reference cDNA; DYNC1LI1, dynein cytoplasmic 1 light intermediate chain 1; CRC, colorectal cancer.

and LS174T. Among these CRC cell lines (AJCC stage II), LS 174T cells had significantly increased expression levels of DYNC1LI1 compared with the HRC control and other CRC cell lines $(\mathrm{P}<0.01$; Fig. 2); therefore, LS 174T cells were used in subsequent experiments. The knockdown efficiency of shDYNC1LI1 in LS 174T cells was subsequently determined using RT-qPCR and western blotting, and it was established to be successful (Fig. 3A and B). Notably, MUC mRNA expression levels were discovered to be differentially altered in the shDYNC1LI1-LS 174T cells (Fig. 3C). Briefly, MUC1 expression levels were significantly decreased by 0.06-fold, whereas the expression levels of MUC2, MUC4 and MUC5AC were all significantly increased by $2.70,4.17$ or 2.75-fold, respectively, in shDYNC1LI1-LS 174T cells compared with the shLUC-LS 174T cells (Fig. 3C). No significant differences were observed in the expression levels of MUC6 between the shDYNC1LI1-LS 174T and shLUC-LS $174 \mathrm{~T}$ cells.

Chemotherapeutic effects in shLUC-LS $174 T$ and shDYNC1LI1-LS 174T cells. 5-FU, leucovorin, and oxaliplatin (FOLFOX4) is a chemotherapeutic reagent used for the treatment of CRC $(24,25)$. It was discovered that the genetic knockdown of DYNC1LI1 in LS 174T cells increased the susceptibility of the cells to 5-FU and oxaliplatin treatment; the $\mathrm{IC}_{50}$ of 5 -FU $(9.7 \mu \mathrm{M})$ and oxaliplatin $(0.4 \mu \mathrm{M})$ was significantly decreased in the shDYNC1LI1-LS 174T cells compared with the shLUC-LS 174T cells $(23.3 \mu \mathrm{M}$ and $1.06 \mu \mathrm{M}$, respectively; Fig. 4A and B). The differences in the cell proliferative rate of shLUC-LS 174T and shDYNC1LI1-LS 174T cells began at day 4 without any drug treatment (Fig. 5A), and at day 3 with 5-FU (Fig. 5B) or oxaliplatin chemotherapy (Fig. 5C). Moreover, the shDYNC1LI1-LS 174T cells exhibited a significantly reduced proliferative rate at day 7 compared with the shLUC-LS 174T cells following both 5-FU or oxaliplatin chemotherapy $(\mathrm{P}<0.05$; Fig. $5 \mathrm{~B}$ and $\mathrm{C})$.

\section{Discussion}

Tumorigenesis involves cytoskeletal reorganization (26) and as demonstrated by van Ree et al (27), cytoplasmic dyneins can regulate centrosome dynamics. Thus, any changes in their function have been hypothesized to lead to cancer formation. For example, it was previously reported that in patients with advanced CRC, the expression levels of the cytoplasmic dynein 1 heavy chain 1 (DYNC1H1) were decreased (28). This aberrant expression of DYNC1H1 was suggested to impede the assembly of complete cytoplasmic dynein, resulting in inadequate microtubule dynamics.

In the present study, increased expression levels of DYNC1LI1 were observed in late-stage, metastatic CRC tissues. Therefore, the tumorigenic potential of DYNC1LI1 in CRC warrants further metastatic investigations and research. The goblet-cell-like LS 174T cells, which exhibit mucinous secretory granules, have previously provided an excellent in vitro model for studying the expression levels of MUCs in CRC (21). The present study revealed that genetic knockdown of DYNC1LI1 induced decreased expression levels of MUC1 and increased expression levels of MUC2, MUC4 and MUC5AC in LS 174T cells. The differentially expressed levels of DYNC1LI1 in LS 174T cells have been suggested to change the MUC profile, thereby generating differences in the mucosal barrier (29). Thus, it can be further hypothesized that DYNC1LI1-induced changes in the mucosal barrier may be positively associated with tumor formation. The integrity of the gut mucosal barrier, which is composed of a specific profile of MUCs, has been found to be crucial for protecting epithelial cells from inflammatory or cancerous responses $(30,31)$.

A previous study provided molecular evidence to indicate that the changes in the expression levels of cytoskeleton-related genes may worsen the prognosis of patients with CRC and lead to the formation of an aberrant mucosal barrier with a different MUC profile (19). A previous study indicated that several MUCs, such as increased MUC1 and decreased MUC2, are associated with CRC (18). Based on the results of the present study and the aforementioned studies, it was hypothesized that increased DYNC1LI1 expression levels may be associated with the advanced stages of CRC. In LS 174T cells, the expression levels of MUC1, a transmembrane glycosylated phosphoprotein, and DYNC1LI1 displayed a similar expression trend in the present study; this result is consistent with that observed in the CRC cell lines, HCT 116 and SW480. A previous study indicated that cells with lower DYNC1LI1 expression levels also had decreased expression levels of MUC1 $(32,33)$. MUC1, a membrane-bound MUC, was also found to be overexpressed in numerous types of human cancer, such as endometrioid endometrial carcinoma and $\mathrm{CaP}(34,35)$. Moreover, patients with increased MUC1 expression levels were suggested to have a poorer prognosis (36). Betge et al (17) concluded that MUC1 expression levels had no effect on the clinical outcomes, although more than half of the studied patients with CRC exhibited MUC1 overexpression. Other previous studies investigating the prognostic value of MUCs in cancers have also reported contradictory results (37-39). These discrepancies in the results of different studies may be attributable to the differences in cytoskeletal gene expression. Thus, DYNC1LI1 
A

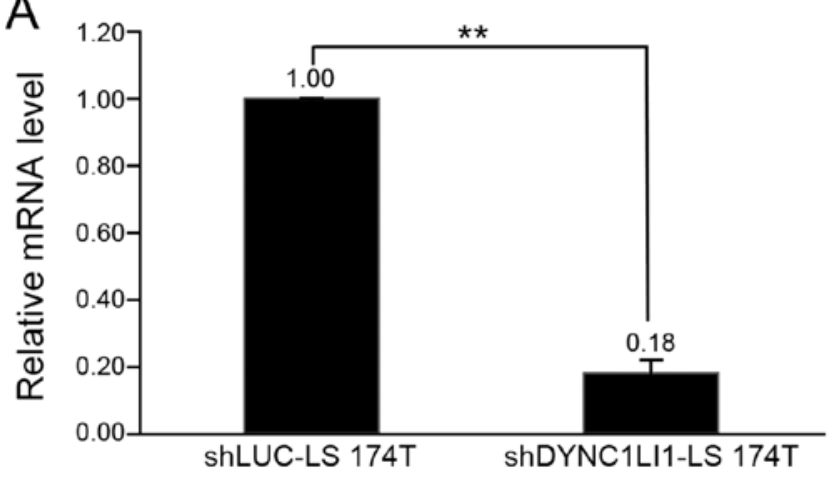

B

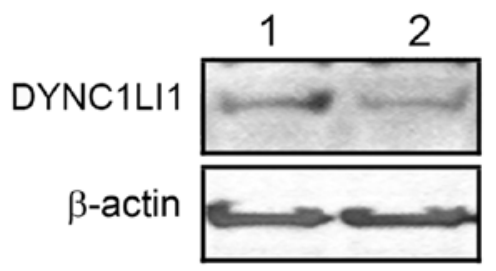

Efficiency of ShDYNC1LI1 in LS 174T cells

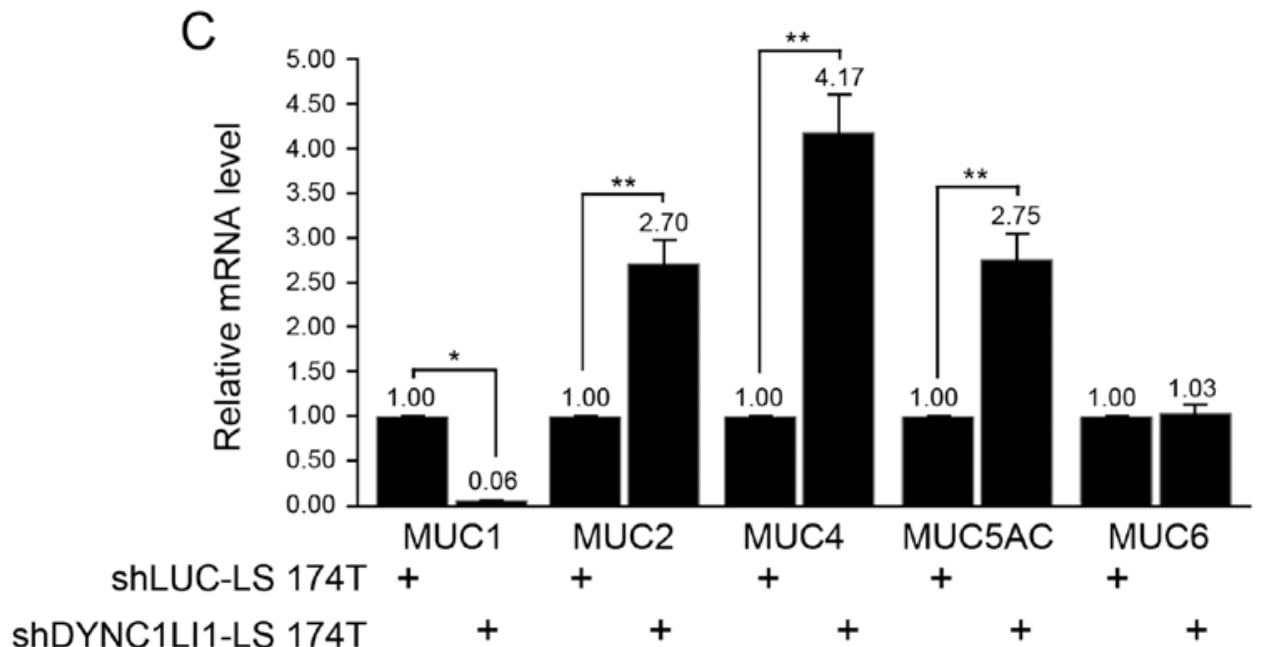

Figure 3. Relative mRNA expression levels of MUCs in shDYNC1LI1-LS 174T cells. (A) Relative mRNA and (B) protein expression levels of DYNC1LI1 were determined by RT-qPCR or western blotting, respectively, in shLUC-LS 174T and shDYNC1LI1-LS 174T cells. (C) Expression levels of MUCs were determined in shLUC-LS 174T and shDYNC1LI1-LS 174T cells. All mRNA expression levels were normalized to GAPDH and protein expression levels were normalized to $\beta$-actin. The Student's t-test was used to determine the efficiency of the shDYNC1LI1 infection in parts A and C. Each experiment was independently performed in duplicate or triplicate. Numerical data are expressed as the mean $\pm \mathrm{SD}$. ${ }^{*} \mathrm{P}<0.05$ and ${ }^{* *} \mathrm{P}<0.01$. MUC, mucin; shRNA, short hairpin RNA; DYNC1LI1, dynein cytoplasmic 1 light intermediate chain 1; LUC, luciferase.

expression levels, and its effects, must be further understood in order to predict patient prognosis.

Secreted gel-forming MUCs, another family of MUCs, include both MUC2 and MUC5AC (18). Betge et al (17) reported that the loss of MUC2 and MUC5AC expression was associated with favorable CRC outcomes; however, only reduced expression levels of MUC2, and not of MUC5AC, were detected in colonic tumors by Byrd and Bresalier (18). Previously, MUC2 was suggested to be a crucial component of the MUC profile in the mucosal barrier, but served no role in carcinogenesis (40). In addition, an animal model indicated that Muc2-knockout mice had a poorly defined mucosal barrier (16). Collectively, these results indicated that DYNC1LI1, which upregulates the expression levels of transmembrane MUCs, such as MUC1, and downregulates the expression levels of secreted gel-forming MUCs, such as MUC2 and MUC5AC, may be critical for assessing the composition of the mucosal barrier in patients with CRC. Compared with normal prostate or benign tissue, $\mathrm{CaP}$ tissue exhibited a similar MUC profile, with increased MUC1 and decreased MUC2, MUC4, MUC5AC and MUC6 expression levels (34). In addition, the difference of immunohistochemical staining patterns of MUC1 and MUC2 were caused by the carcinomatous transformation of urothelial neoplastic and preneoplastic lesions (41). Taken together, these findings suggested that different types of cancer, including those of the urological and gastrointestinal systems, exhibit diverse MUC profiles.

However, the possibility that disrupting the microtubule dynamics in shDYNC1LI1-LS 174T cells may influence cell proliferation cannot be excluded. For example, Even et al (42) reported that DYNC1LI1 contributed to the proliferative overgrowth of CRC cells, which is consistent with the present study results, which found that shDYNC1LI1-LS 174T cells had a slower proliferative rate compared with shLUC-LS 174T cells without any drug treatment. Adjuvant chemotherapy, such as 5-FU or oxaliplatin, which are the ingredients of FOLFOX4, was discovered to further inhibit the proliferation of LS 174T cells with low DYNC1LI1 expression levels. Thus, these findings suggested that DYNC1LI1 may be a marker molecule for prescribing 5-FU as a first-line chemotherapy treatment for patients with CRC. In our in vitro cell study, shDYNC1LI1-LS 

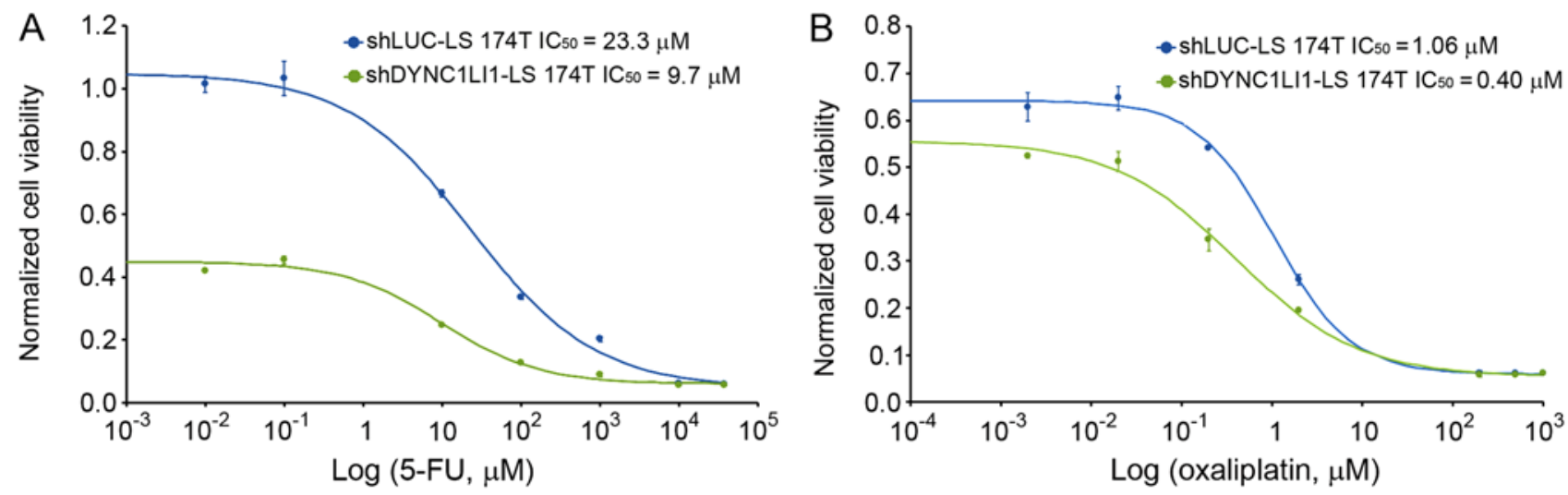

Figure 4. Effect of DYNC1LI1 on the chemosensitivity of LS 174T cells. Chemosensitivity of shDUNC1LI1-LS 174T and shLUC-LS 174T cells to (A) 5-FU and (B) oxaliplatin. shLUC-LS 174T (blue line) and shDYNC1LI1-LS 174T (green line) cells were independently treated with the indicated concentrations of 5-FU or oxaliplatin for $72 \mathrm{~h}$. The MTT assay was used to determine the cell viability of cells in culture. The data line represents the best-fit curve and $\mathrm{IC}_{50}$ calculations were performed with a computer program using curve interpolation (four-parameter logistics), Gen5 ${ }^{\mathrm{TM}}$ Data Analysis. 5-FU, 5-fluorouracil; $\mathrm{IC}_{50}$, the half maximal inhibitory concentration; shRNA, short hairpin RNA; DYNC1LI1, dynein cytoplasmic 1 light intermediate chain 1; LUC, luciferase.

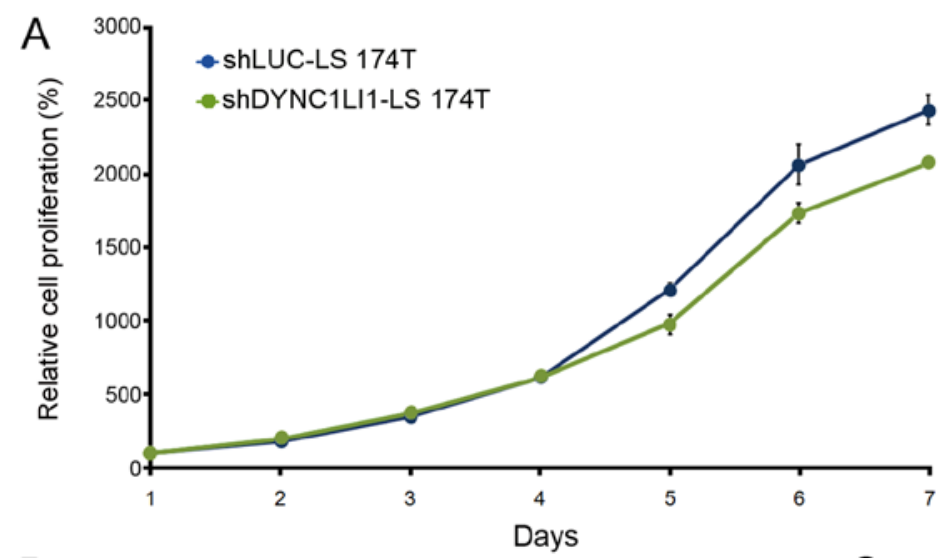

\section{B}

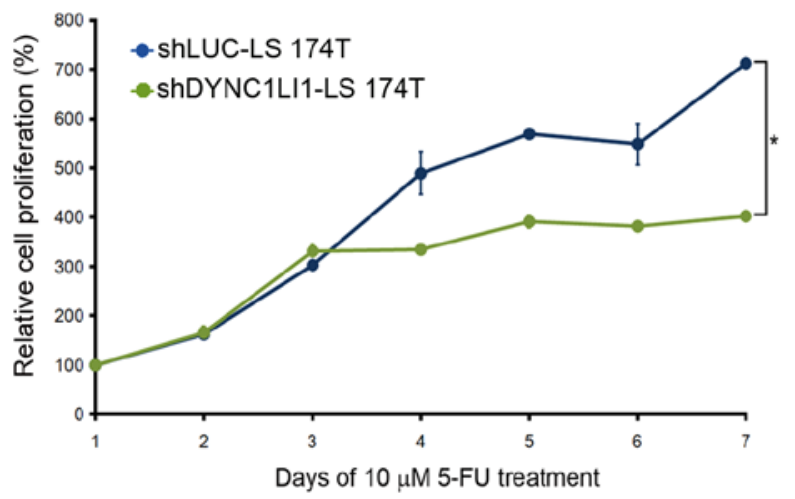

C

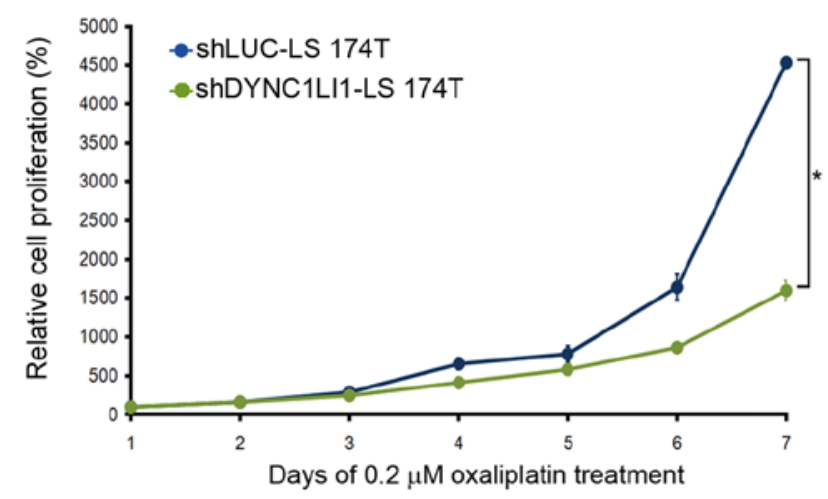

Figure 5. Effect of DYNC1LI1 on the cell proliferative rate of LS 174T cells in response to different chemotherapeutic drugs. Cell proliferative rate of shDUNC1LI1-LS 174T and shLUC-LS 174T cells was determined (A) without any drug treatment, (B) with $10 \mu \mathrm{M} 5$-FU treatment and (C) with $0.2 \mu \mathrm{M}$ oxaliplatin treatment. The proliferative rate of shLUC-LS 174T and shDYNC1LI1-LS 174T cells was evaluated using the MTT assay. Each experimental condition was repeated in duplicate or triplicate. The Student's t-test was used to compare the two independent groups. Numerical data are expressed as the mean \pm SD. ${ }^{*} \mathrm{P}<0.05 .5-\mathrm{FU}, 5$-fluorouracil; shRNA, short hairpin RNA; DYNC1LI1, dynein cytoplasmic 1 light intermediate chain 1; LUC, luciferase.

$174 \mathrm{~T}$ cells were more sensitive to 5-FU treatment compared with cells with endogenous DYNC1LI1 expression levels. As previously discussed, shDYNC1LI1-LS 174T cells had low MUC1, and high MUC2, MUC4 and MUC5AC expression levels, implying that CRC cells with low MUC1 expression levels and high expression levels of other MUCs may benefit from 5-FU-based therapy. Furthermore, this result was similar to findings in pancreatic cancer cells reported by Kalra and Campbell (43), where it was demonstrated that increased MUC1 expression levels potentially impeded the cytotoxic effects of 5-FU against the proliferation of pancreatic cancer cells.

In conclusion, based on the findings of the present results and those of other previous studies, it was suggested that DYNC1LI1, which is significantly associated with MUC 
expression levels, may affect the efficiency of chemotherapy. Additional functional studies and clinical reports are required for understanding its molecular significance in tumorigenesis.

\section{Acknowledgements}

Not applicable.

\section{Funding}

The current study was supported by grants from the Cathay General Hospital and Taipei Medical University (grant nos. 104CGH-TMU-05 and 105CGH-TMU-04) and the Cathay General Hospital (grant no. CGH-MR-A10316).

\section{Availability of data and materials}

The datasets used and/or analyzed during the present study are available from the corresponding author on reasonable request.

\section{Authors' contributions}

CCC and KCC performed the experiments and wrote the manuscript. CJH performed the statistical analysis and helped revise the manuscript. $\mathrm{CSH}$ contributed to the interpretation of the data. YCW conceived and designed the study. All authors read and approved the final manuscript.

\section{Ethics approval and consent to participate}

Not applicable.

\section{Patient consent for publication}

Not applicable.

\section{Competing interests}

The authors declare that they have no competing interests.

\section{References}

1. Singh R, Kapur N, Mir H, Singh N, Lillard JW Jr and Singh S: CXCR6-CXCL16 axis promotes prostate cancer by mediating cytoskeleton rearrangement via Ezrin activation and alphavbeta 3 integrin clustering. Oncotarget 7: 7343-7353, 2016.

2. Wu K, Zhang X, Li F, Xiao D, Hou Y, Zhu S, Liu D, Ye X, Ye M, Yang J, et al: Frequent alterations in cytoskeleton remodelling genes in primary and metastatic lung adenocarcinomas. Nat Commun 6: 10131, 2015.

3. Kuijpers M, van de Willige D, Freal A, Chazeau A, Franker MA, Hofenk J, Rodrigues RJ, Kapitein LC, Akhmanova A, Jaarsma D and Hoogenraad CC: Dynein regulator NDEL1 controls polarized cargo transport at the axon initial segment. Neuron 89: 461-471, 2016.

4. McKenney RJ, Huynh W, Tanenbaum ME, Bhabha G and Vale RD: Activation of cytoplasmic dynein motility by dynactin-cargo adapter complexes. Science 345: 337-341, 2014.

5. McLaughlin RT, Diehl MR and Kolomeisky AB: Collective dynamics of processive cytoskeletal motors. Soft Matter 12: $14-21,2015$.

6. Johnson JL, Hamm-Alvarez S, Payne G, Sancar GB, Rajagopalan KV and Sancar A: Identification of the second chromophore of Escherichia coli and yeast DNA photolyases as 5,10-methenyltetrahydrofolate. Proc Natl Acad Sci USA 85 2046-2050, 1988.
7. Schnaeker EM, Ossig R, Ludwig T, Dreier R, Oberleithner H, Wilhelmi M and Schneider SW: Microtubule-dependent matrix metalloproteinase-2/matrix metalloproteinase-9 exocytosis: Prerequisite in human melanoma cell invasion. Cancer Res 64: 8924-8931, 2004.

8. Vidulescu C, Clejan S and O'Connor KC: Vesicle traffic through intercellular bridges in DU 145 human prostate cancer cells. J Cell Mol Med 8: 388-396, 2004.

9. Yoon SO, Shin S and Mercurio AM: Hypoxia stimulates carcinoma invasion by stabilizing microtubules and promoting the Rab11 trafficking of the alpha6beta4 integrin. Cancer Res 65: 2761-2769, 2005.

10. Izidoro-Toledo TC, Borges AC, Araujo DD, Mazzi DP, Nascimento Junior FO, Sousa JF, Alves CP, Paiva AP, Trindade DM, Patussi EV, et al: A myosin-Va tail fragment sequesters dynein light chains leading to apoptosis in melanoma cells. Cell Death Dis 4: e547, 2013.

11. Jiang J, Yu L, Huang X, Chen X, Li D, Zhang Y, Tang L and Zhao S: Identification of two novel human dynein light chain genes, DNLC2A and DNLC2B, and their expression changes in hepatocellular carcinoma tissues from 68 Chinese patients. Gene 281: 103-113, 2001.

12. Dong H, Zhang H, Liang J, Yan H, Chen Y, Shen Y, Kong Y, Wang S, Zhao G and Jin W: Digital karyotyping reveals probable target genes at 7q21.3 locus in hepatocellular carcinoma. BMC Med Genomics 4: 60, 2011.

13. Weeks ME, Hariharan D, Petronijevic L, Radon TP, Whiteman HJ, Kocher HM, Timms JF, Lemoine NR and Crnogorac-Jurcevic T: Analysis of the urine proteome in patients with pancreatic ductal adenocarcinoma. Proteomics Clin Appl 2: 1047-1057, 2008.

14. Zhao H, Pflug BR, Lai X and Wang M: Pyruvate dehydrogenase alpha 1 as a target of omega-3 polyunsaturated fatty acids in human prostate cancer through a global phosphoproteomic analysis. Proteomics 16: 2419-2431, 2016.

15. Parsons CL: The role of the urinary epithelium in the pathogenesis of interstitial cystitis/prostatitis/urethritis. Urology 69: 9-16, 2007.

16. Petersson J, Schreiber O, Hansson GC, Gendler SJ, Velcich A, Lundberg JO, Roos S, Holm L and Phillipson M: Importance and regulation of the colonic mucus barrier in a mouse model of colitis Am J Physiol Gastrointest Liver Physiol 300: G327-G333, 2011.

17. Betge J, Schneider NI, Harbaum L, Pollheimer MJ, Lindtner RA, Kornprat P, Ebert MP and Langner C: MUC1, MUC2, MUC5AC, and MUC6 in colorectal cancer: Expression profiles and clinical significance. Virchows Arch 469: 255-265, 2016.

18. Byrd JC and Bresalier RS: Mucins and mucin binding proteins in colorectal cancer. Cancer Metastasis Rev 23: 77-99, 2004.

19. Kufe DW: Mucins in cancer: function, prognosis and therapy. Nat Rev Cancer 9: 874-885, 2009.

20. Ivanov AI, Parkos CA and Nusrat A: Cytoskeletal regulation of epithelial barrier function during inflammation. Am J Pathol 177: 512-524, 2010.

21. Bu XD, Li N, Tian XQ and Huang PL: Caco-2 and LS174T cell lines provide different models for studying mucin expression in colon cancer. Tissue Cell 43: 201-206, 2011.

22. Du J, Zheng X, Cai S, Zhu Z, Tan J, Hu B, Huang Z and Jiao H: MicroRNA506 participates in pancreatic cancer pathogenesis by targeting PIM3. Mol Med Rep 12: 5121-5126, 2015.

23. Ohuchida K, Mizumoto K, Yamada D, Fujii K, Ishikawa N, Konomi H, Nagai E, Yamaguchi K, Tsuneyoshi M and Tanaka M: Quantitative analysis of MUC1 and MUC5AC mRNA in pancreatic juice for preoperative diagnosis of pancreatic cancer. Int J Cancer 118: 405-411, 2006.

24. Baratelli C, Zichi C, Di Maio M, Brizzi MP, Sonetto C, Scagliotti GV and Tampellini M: A systematic review of the safety profile of the different combinations of fluoropyrimidines and oxaliplatin in the treatment of colorectal cancer patients. Crit Rev Oncol Hematol 122: 21-29, 2018.

25. Park SH, Sung JY, Han SH, Baek JH, Oh JH, Bang SM, Cho EK, Shin DB and Lee JH: Oxaliplatin, folinic acid and 5-fluorouracil (FOLFOX-4) combination chemotherapy as second-line treatment in advanced colorectal cancer patients with irinotecan failure: A Korean single-center experience. Jpn J Clin Oncol 35: 531-535, 2005.

26. Fearnhead NS, Wilding JL and Bodmer WF: Genetics of colorectal cancer: Hereditary aspects and overview of colorectal tumorigenesis. Br Med Bull 64: 27-43, 2002.

27. van Ree JH, Nam HJ and van Deursen JM: Mitotic kinase cascades orchestrating timely disjunction and movement of centrosomes maintain chromosomal stability and prevent cancer. Chromosome Res 24: 67-76, 2016. 
28. Chang CC, Chien CC, Yang SH, Chen SH and Huang CJ: Identification and clinical correlation of decreased expression of cytoplasmic dynein heavy chain 1 in patients with colorectal cancer. Clin Mol Medicine 1: 6-10, 2008.

29. Holmberg FEO, Pedersen J, Jorgensen P, Soendergaard C, Jensen $\mathrm{KB}$ and Nielsen $\mathrm{OH}$ : Intestinal barrier integrity and inflammatory bowel disease: Stem cell-based approaches to regenerate the barrier. J Tissue Eng Regen Med 12: 923-935, 2018.

30. Gouyer V, Dubuquoy L, Robbe-Masselot C, Neut C, Singer E Plet S, Geboes K, Desreumaux P, Gottrand F and Desseyn JL: Delivery of a mucin domain enriched in cysteine residues strengthens the intestinal mucous barrier. Sci Rep 5: 9577, 2015.

31. Tadesse S, Corner G, Dhima E, Houston M, Guha C, Augenlicht L and Velcich A: MUC2 mucin deficiency alters inflammatory and metabolic pathways in the mouse intestinal mucosa Oncotarget 8: 71456-71470, 2017.

32. McAuley JL, Linden SK, Png CW, King RM, Pennington HL, Gendler SJ, Florin TH, Hill GR, Korolik V and McGuckin MA: MUC1 cell surface mucin is a critical element of the mucosal barrier to infection. J Clin Invest 117: 2313-2324, 2007.

33. Ren J, Agata N, Chen D, Li Y, Yu WH, Huang L, Raina D, Chen W, Kharbanda S and Kufe D: Human MUC1 carcinoma-associated protein confers resistance to genotoxic anticancer agents. Cancer Cell 5: 163-175, 2004.

34. Cozzi PJ, Wang J, Delprado W, Perkins AC, Allen BJ, Russell PJ and Li Y: MUC1, MUC2, MUC4, MUC5AC and MUC6 expression in the progression of prostate cancer. Clin Exp Metastasis 22: 565-573, 2005.

35. Morrison C, Merati K, Marsh WL Jr, De Lott L, Cohn DE, Young G and Frankel WL: The mucin expression profile of endometrial carcinoma and correlation with clinical-pathologic parameters. Appl Immunohistochem Mol Morphol 15: 426-431, 2007.

36. Handra-Luca A, Lamas G, Bertrand JC and Fouret P: MUC1, MUC2, MUC4, and MUC5AC expression in salivary gland mucoepidermoid carcinoma: Diagnostic and prognostic implications. Am J Surg Pathol 29: 881-889, 2005.
37. Hosseini S, Bananzadeh AM, Salek R, Zare-Bandamiri M, Kermani AT and Mohammadianpanah M: Prognostic significance of mucinous histologic subtype on oncologic outcomes in patients with colorectal cancer. Ann Coloproctol 33: 57-63, 2017.

38. Purdie CA and Piris J: Histopathological grade, mucinous differentiation and DNA ploidy in relation to prognosis in colorectal carcinoma. Histopathology 36: 121-126, 2000.

39. Xie L, Villeneuve PJ and Shaw A: Survival of patients diagnosed with either colorectal mucinous or non-mucinous adenocarcinoma: A population-based study in Canada. Int J Oncol 34: 1109-1115, 2009.

40. Niv Y and Rokkas T: Mucin expression in colorectal cancer(CRC): Systematic review and meta-analysis. J Clin Gastroenterol 53: 434-440, 2018

41. Gonul II, Cakir A and Sozen S: Immunohistochemical expression profiles of MUC1 and MUC2 mucins in urothelial tumors of bladder. Indian J Pathol Microbiol 61: 350-355, 2018.

42. Even I, Reidenbach S, Schlechter T, Berns N, Herold R, Roth W, Krunic D, Riechmann V and Hofmann I: DLIC1, but not DLIC2, is upregulated in colon cancer and this contributes to proliferative overgrowth and migratory characteristics of cancer cells. FEBS J 286: 803-820, 2019.

43. Kalra AV and Campbell RB: Mucin impedes cytotoxic effect of 5-FU against growth of human pancreatic cancer cells: Overcoming cellular barriers for therapeutic gain. Br J Cancer 97: 910-918, 2007.

This work is licensed under a Creative Commons Attribution-NonCommercial-NoDerivatives 4.0 International (CC BY-NC-ND 4.0) License. 